\title{
puente D̊lend Suecia
}

SKÃNSKA CEMENTGJUTERIET

$562 \cdot 139$

\section{sinopsis}

El puente de Oland salva el estrecho de Kalmar, partiendo de Möll. storpsläge, en la isla de Óland, hasta la isla de N. Skallo: de aqui a la de Svino y desde esta hasta Jutnabben. en la peninsula sueca. Tiene 155 vanos: 147 de luces pequeñas, 2 de luces medianas y 6 centrales. de $130 \mathrm{~m}$ cada una, con una longitud total de $6.070 \mathrm{~m}$.

Los arcos denominados altos se han realizado por el sistema de voladizos sucesivos, y los bajos, mediante lanzamiento de grandes vigas de hormigón armado. Cimientos sobre pilotes o vertidos in situ, en cajones vaciados neumáticamente, según los casos, y pilas de hormigón armado, construidas con auxilio de encofrados deslizantes.

La Empresa constructora ha sido Skånska Cementgjuteriet, por cuenta de la Administración del Estado. 


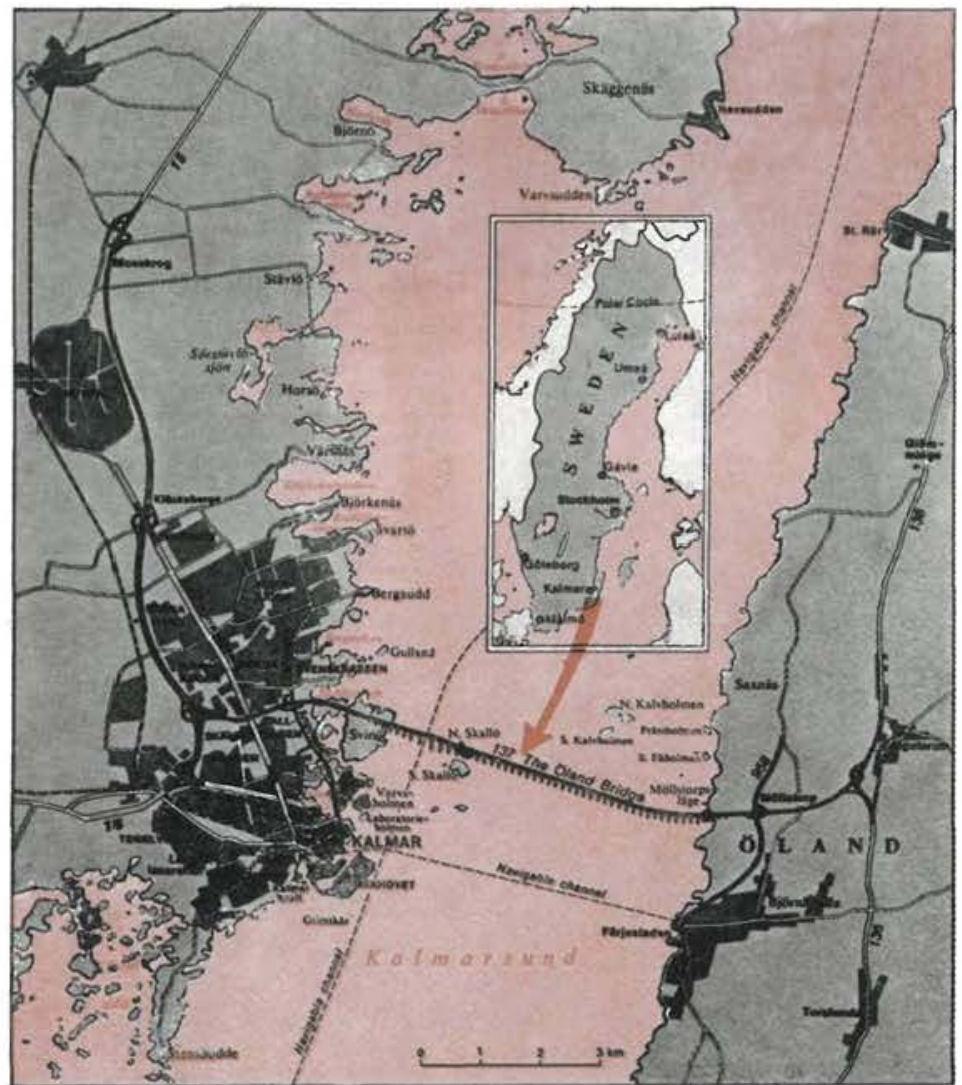

El aumento de la corriente turística desde el Continente hacia la atractiva isla de Öland - panorámicas, fauna, excavaciones

arqueológicas y multitud de molinos de viento-, que estaba abastecido por frecuentes viajes en barco, exigió la construcción de una arteria de circulación rápida cuyo tramo más importante es el puente que aquí se describe, sobre el estrecho de Kalmar. La vía parte de Tallhagen, salta a la isla de Jutnabben; de ésta, a la de Svinö; de aquí, a la de Skallö Norte y, finalmente, a la de Öland.

El puente consta de 155 tramos y es el más largo de Europa, con sus $6 \mathrm{~km}$ de longitud total, distribuyéndose en tres partes principales:

El viaducto de acceso oeste, compuesto por 23 tramos de $34,57 \mathrm{~m}$ cada uno de luz, de los cuales 20 están sobre la isla de Svinö y los tres restantes sobre el agua.

El puente principal, que consta de 6 tramos intermedios de $130 \mathrm{~m}$ y 2 extremos de $65 \mathrm{~m}$.

\section{situación}

El viaducto de acceso este, distribuido en 124 luces de $35,12 \mathrm{~m}$ cada una.

En general está concebido como un gran tramo continuo de hormigón armado, con los tableros de los tramos de acceso formados por vigas de sección constante, uniendo las diferentes pilas, a excepción del puente principal, constituido por siete dobles ménsulas de hormigón pretensado, con juntas, en la mitad de cada gran luz, a $65 \mathrm{~m}$ de las pilas adyacentes respectivas.

En planta la obra presenta un desarrollo rectilíneo de $4,800 \mathrm{~km}$, con una parte curva, cerca de la isla de Öland, cuya longitud es de $1,200 \mathrm{~km}$ y el radio de curvatura de $4 \mathrm{~km}$.

La altura de las pilas es sumamente variable, teniendo en cuenta las condicionantes imperativas de: pendientes razonables de tráfico superior de vehículos, paso inferior de bloques de hielo que las corrientes del Kalmar Sund arrastra periódicamente y la exigencia de la navegación inferior. La altura libre máxima se alcanza entre las pilas 28 y 29 , con $36 \mathrm{~m}$ sobre el nivel del agua en una anchura de $80 \mathrm{~m}$. 
parte baja

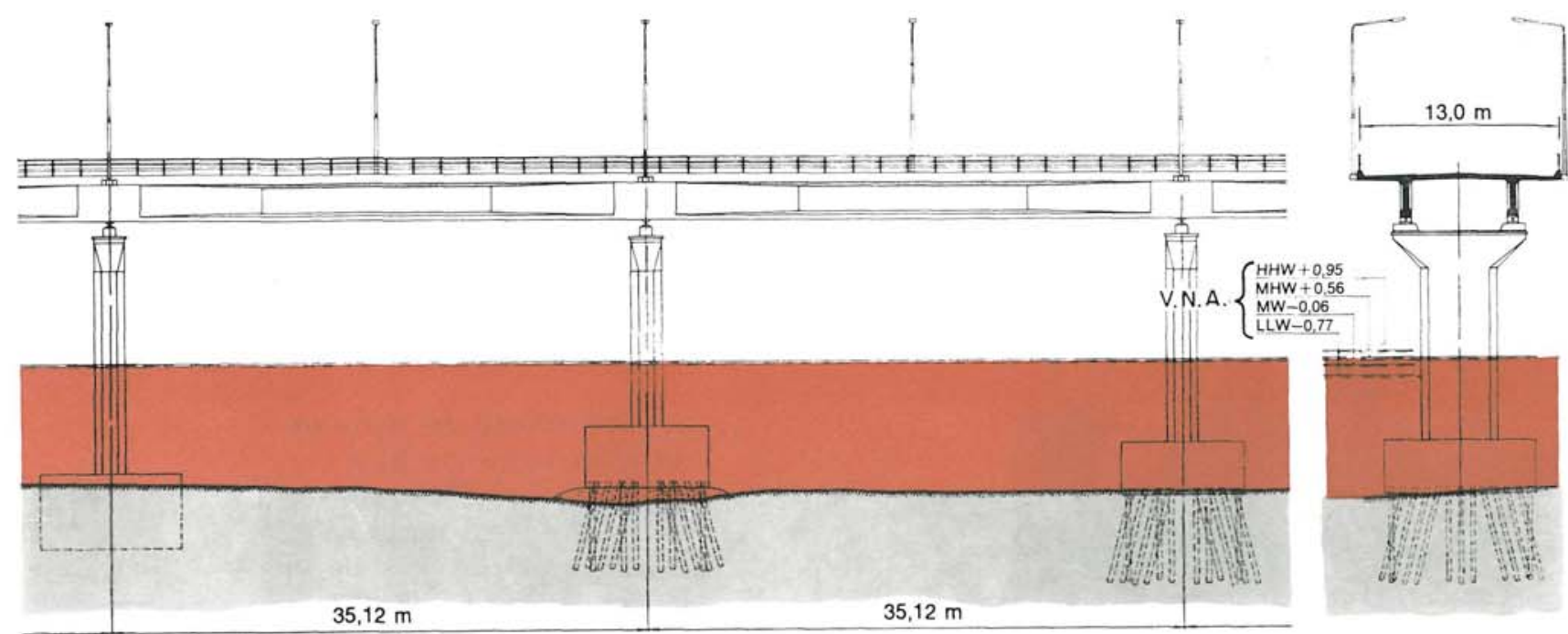

alzado

sección

rectos y transversalmente se tiene una pendiente doble del $2 \%$, desde el eje longitudinal hacia los bordes: en los tres tramos curvos. esta pendiente es también del $2 \%$, pero uniformemente hacia el centro de la curvatura adoptada.

El fondo rocoso se encontró, generalmente, a demasiada profundidad para ser utilizado como apoyo de los cimientos, de modo que sólo

Las condiciones de cimentación se estudiaron cuidadosamente y se manifestaron como extraordinariamente

La anchura total del tablero es de $13,62 \mathrm{~m}$, con una anchura útil de $13 \mathrm{~m}$, formada por una zona central de $7 \mathrm{~m}$ y dos voladizos de $3 \mathrm{~m}$ cada uno. En los tramos variables, dominando los fondos limosos, con zonas morrénicas y sedimentos irregularmente distribuidos. en alguna pilas, sobre la isla de Skällo Norte, pudo cimentarse directamente sobre la roca.

Desde el punto de vista hidráulico, los problemas más importantes surgieron de la presencia de formaciones

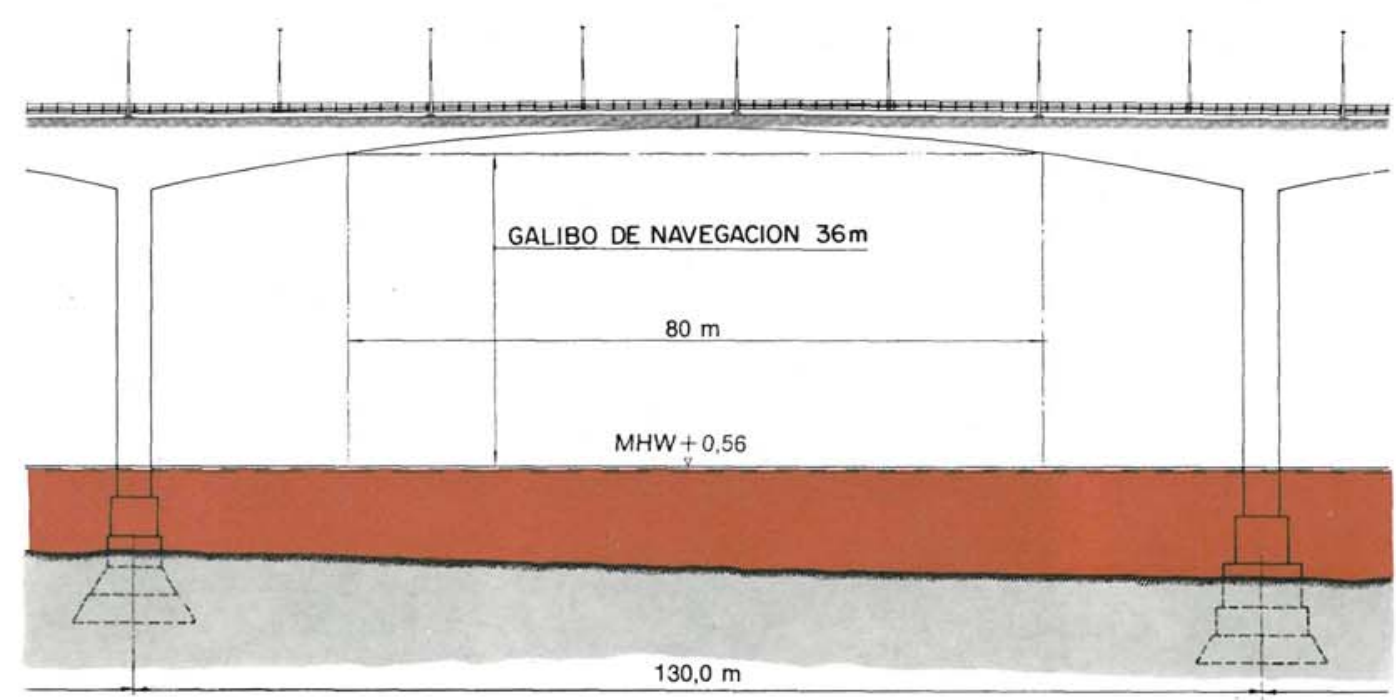

parte alta

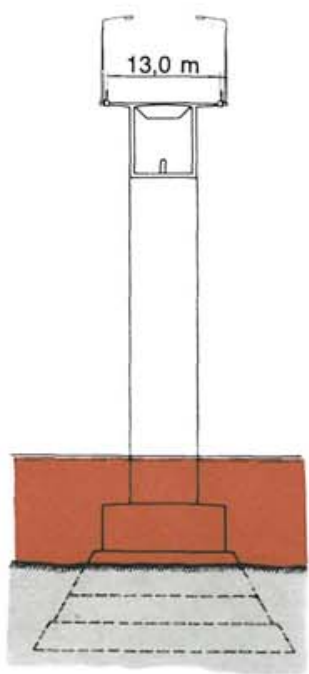

sección 

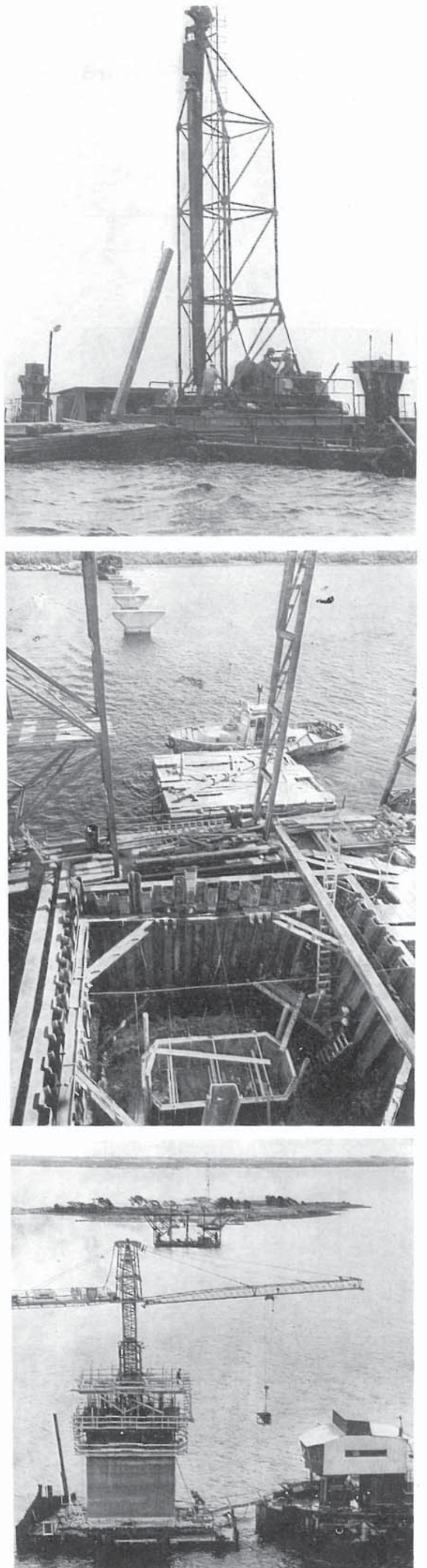

(C) Consejo Superior de Investigaciones Científicas Licencia Creative Commons 3.0 España (by-nc) de hielo, que alcanzan los $10 \mathrm{~m}$ de altura y cubren la totalidad del estrecho de Kalmar, durante varios meses, creando presiones considerables que condicionaron el proyecto de la estructura y especialmente de las pilas.

La profundidad del agua es de $12 \mathrm{~m}$, entre las islas de Svinö y de Skällo Norte; de $10 \mathrm{~m}$, cerca de la de öland, y el resto es de una media de $5 \mathrm{~m}$. La diferencia del nivel del agua entre la marea alta y la baja es de $1,70 \mathrm{~m}$, y las olas máximas alcanzan los $1,50 \mathrm{~m}$.

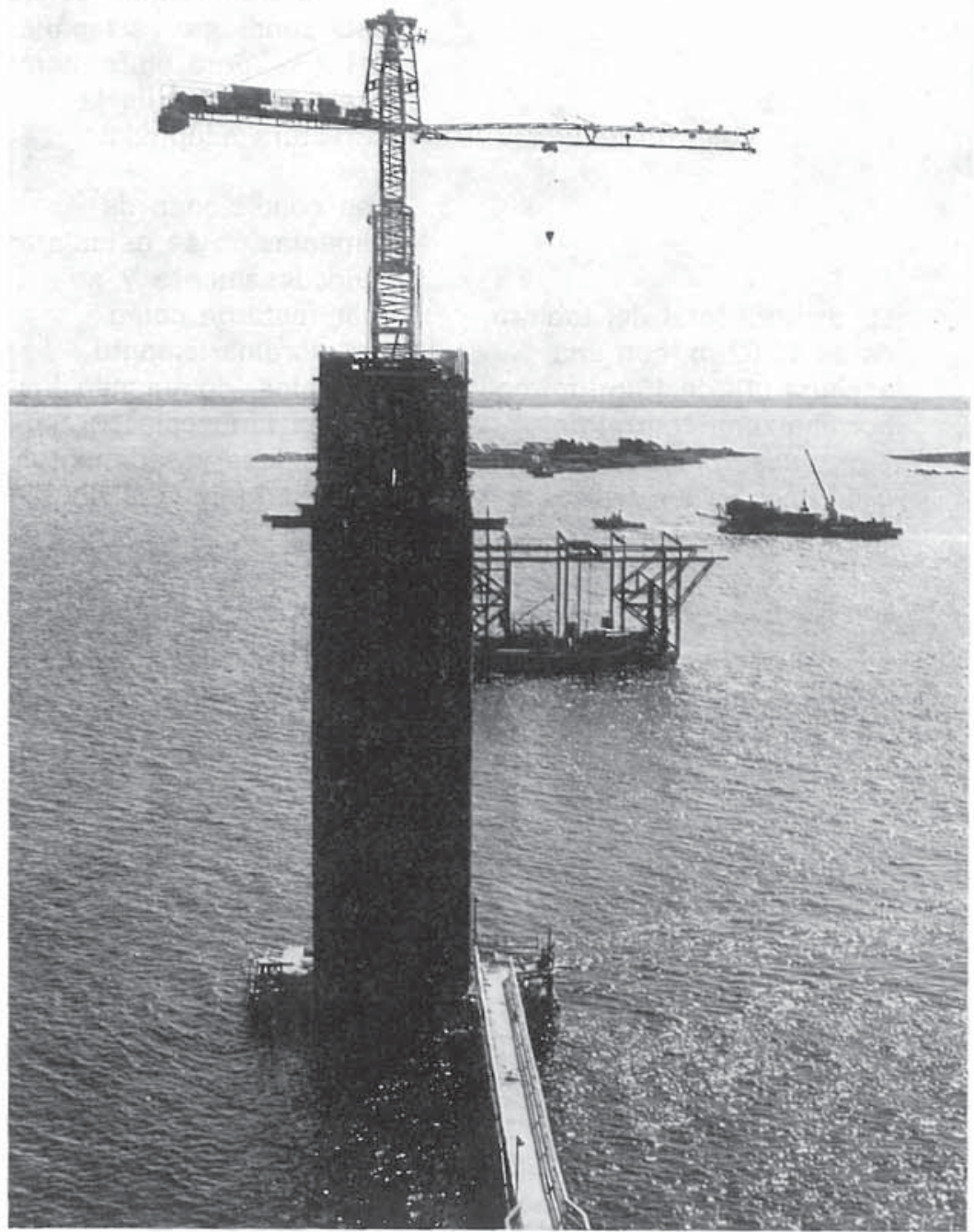




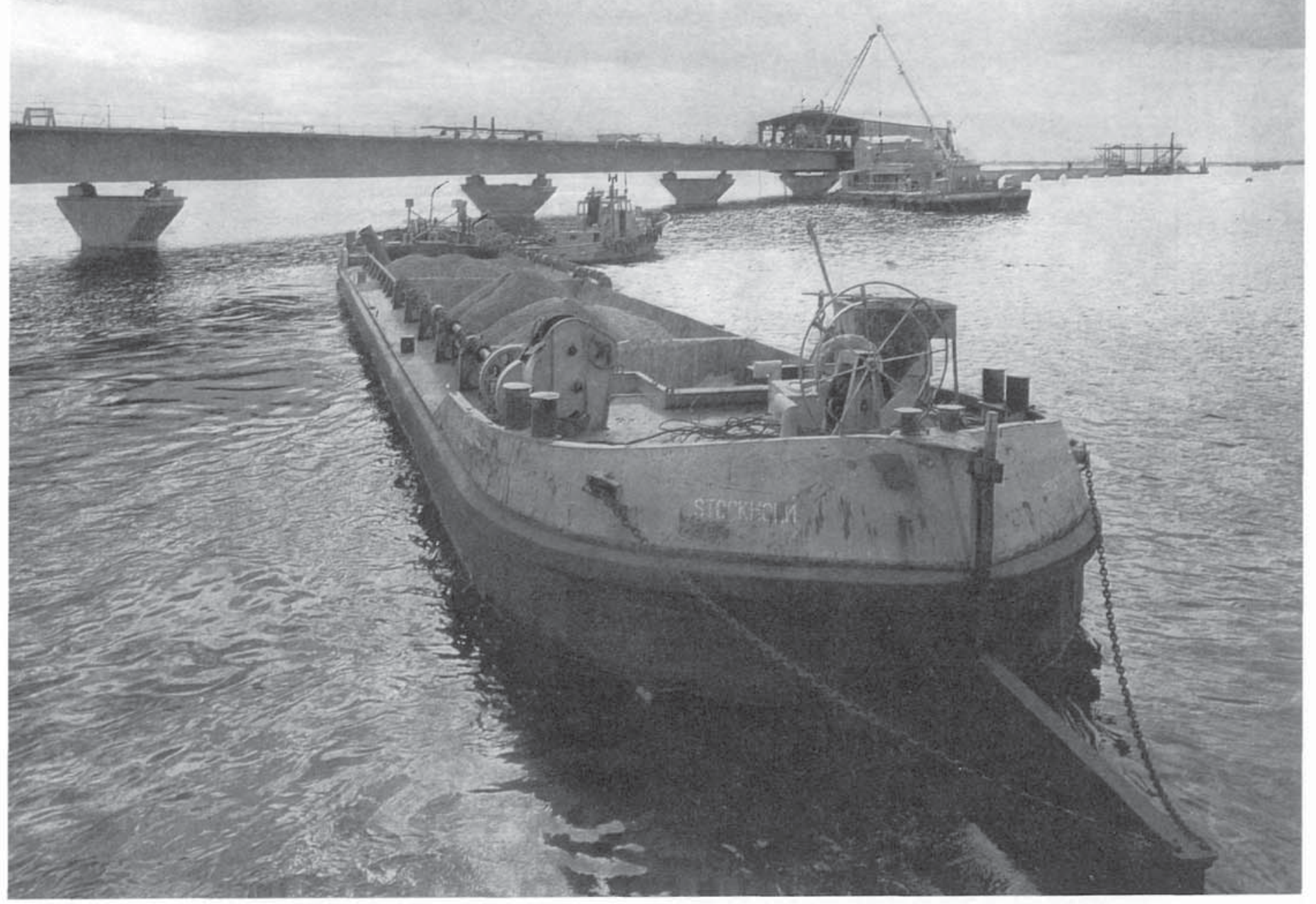

Asimismo, las corrientes son bastante rápidas, entre los 3 y los 7 nudos, lo cual condicionó fuertemente la estructura adoptada, sobre todo en la parte más castigada por esta circunstancia, que era la correspondiente al viaducto de acceso este.

armaduras parte inferior viga tipo

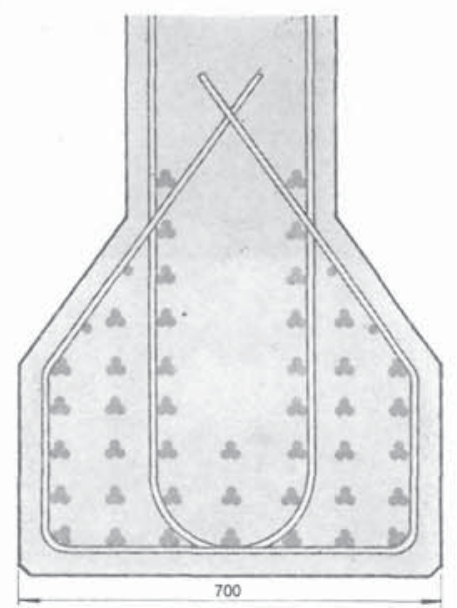

ANCHURA LIBRE $13.0 \mathrm{~m}$

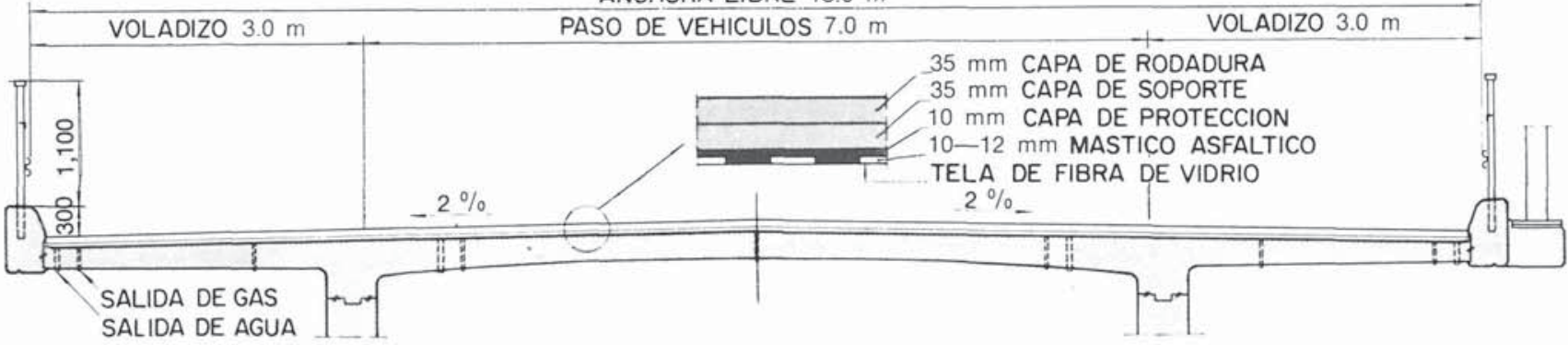

sección transversal 

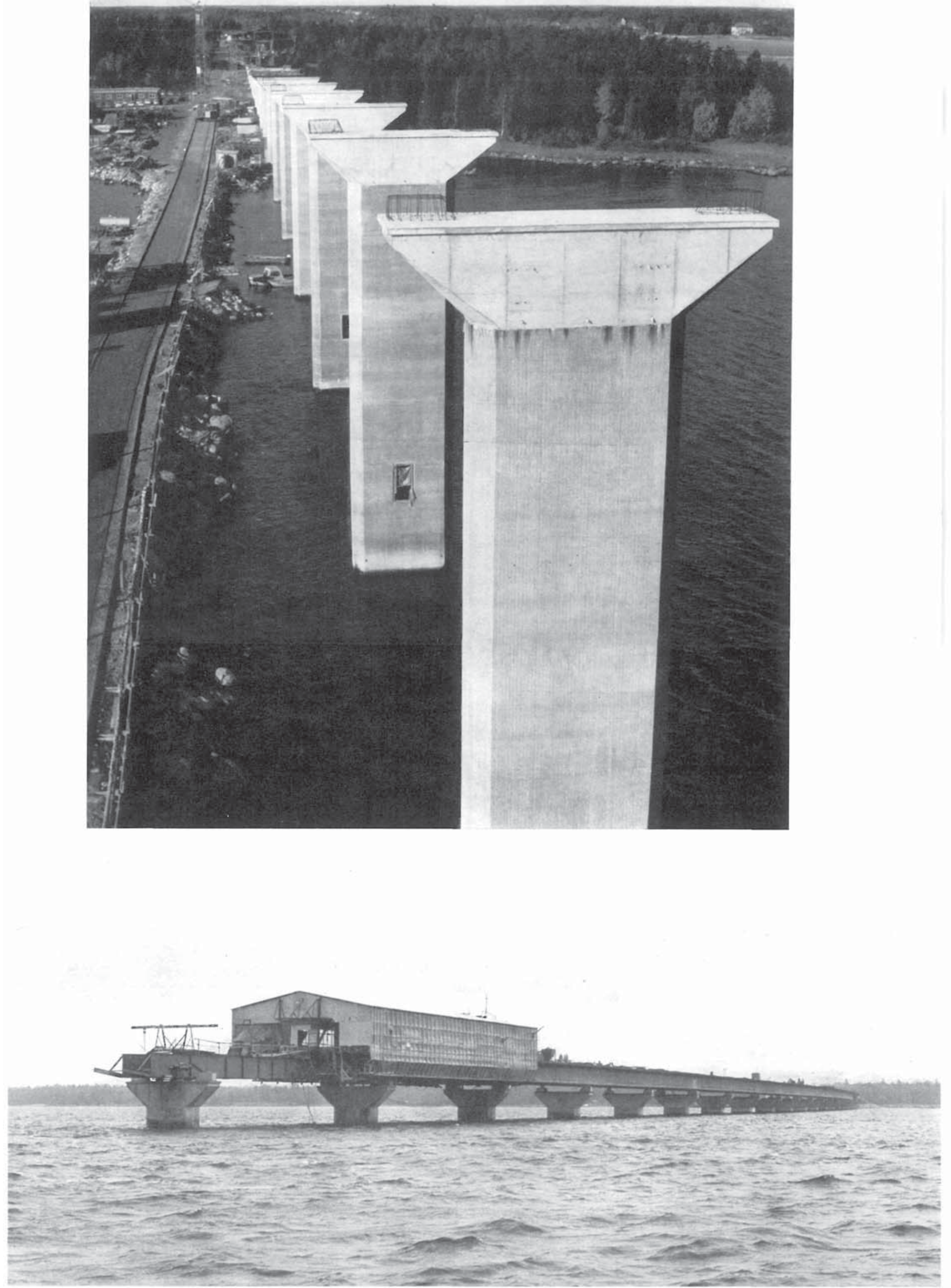


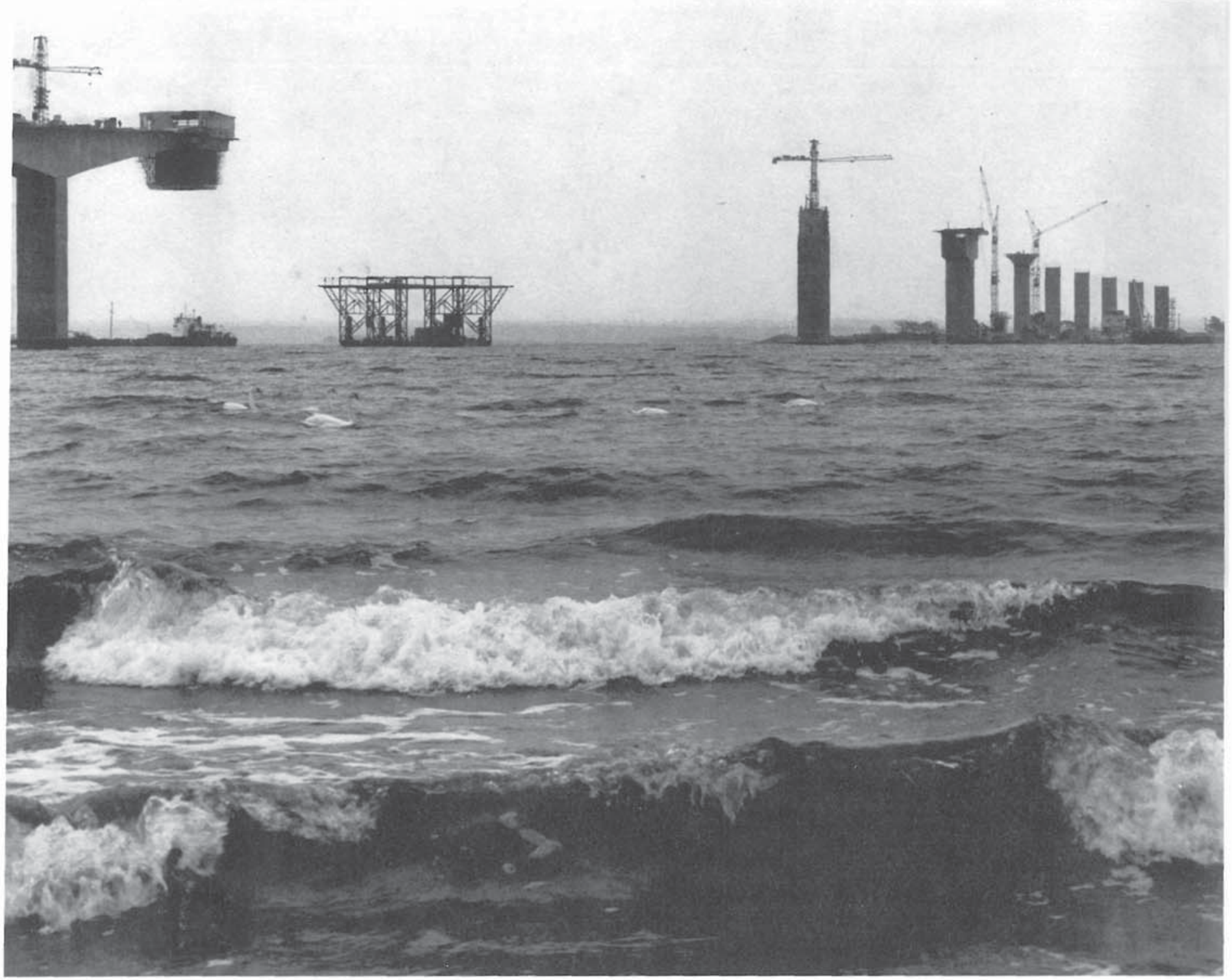

El puente principal

comprende -como ya

dijimos - los tramos entre

las pilas 24 y 32 . Las 5 pilas,

26 a 30 , sobre las que

apoyan las ménsulas dobles,

están cimentadas sobre

planos, el más profundo

de los cuales alcanza

los $-26 \mathrm{~m}$, si bien $14 \mathrm{de}$ ellos son de agua. La anchura del rectángulo de apoyo es, en todas, de $15 \mathrm{~m}$, con longitudes variables de 23 a $30 \mathrm{~m}$.

Las 5 pilas tienen $4 \times 7,8 \mathrm{~m}$ en planta, con refuerzos de $0,15 \times 0,40 \mathrm{~m}$ en las esquinas, para protección contra los bloques de hielo.

Las ménsulas presentan altura variable, desde $8,52 \mathrm{~m} \quad$ y fondo variable de 18 en el arranque a $1,80 \mathrm{~m}$ en la clave, con longitud de $7,70 \mathrm{~m}$. Son de sección cajón (paredes de $45 \mathrm{~cm}$ a $52 \mathrm{~cm}$ ) y fueron

pretensadas con cables

Dywidag $\varnothing 26 \mathrm{~mm}$, en número máximo de 276. 

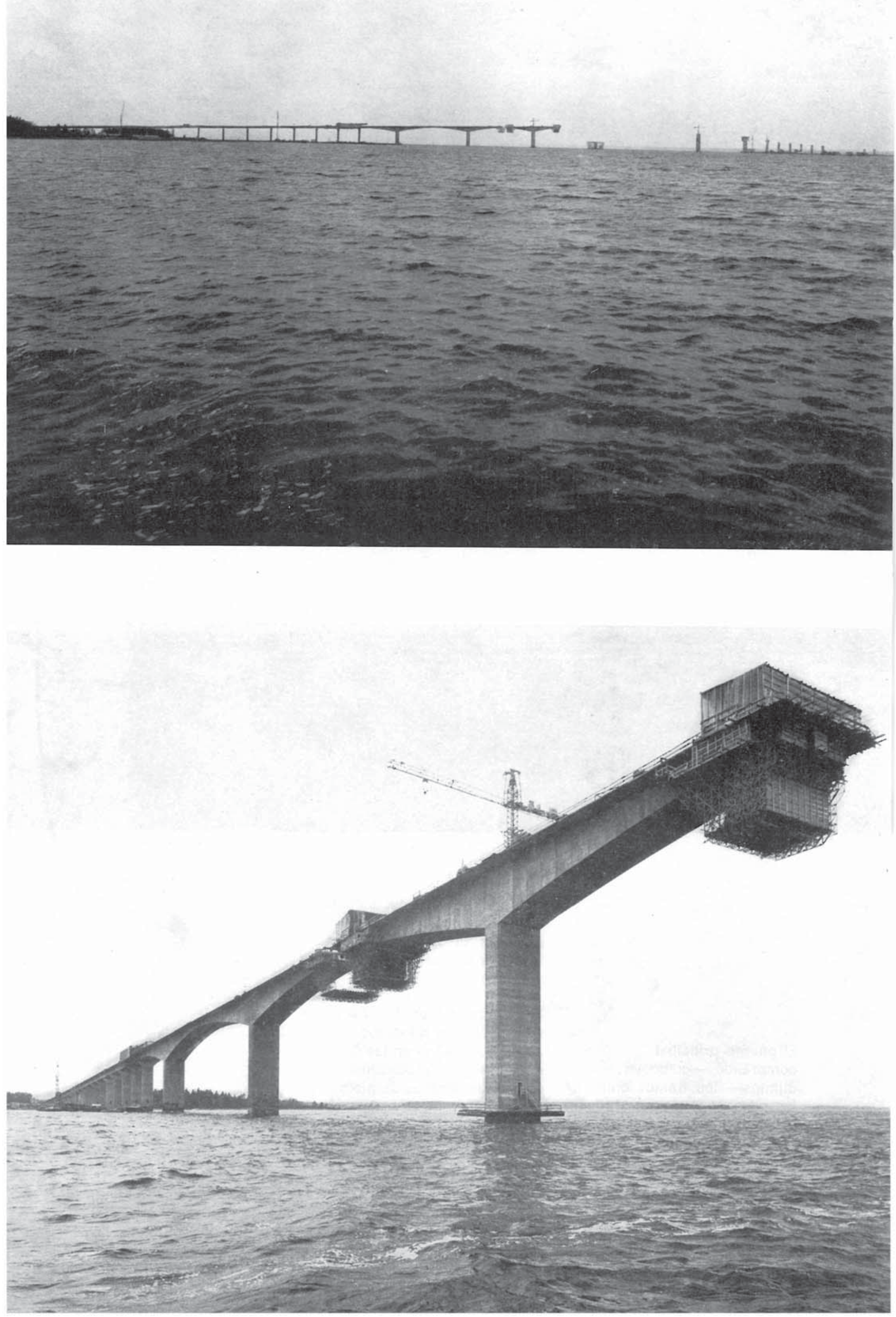

(C) Consejo Superior de Investigaciones Científicas Licencia Creative Commons 3.0 España (by-nc) 
Las pilas de los viaductos de acceso tienen $2 \times 2 \mathrm{~m}$ en planta, con refuerzos de $0,50 \times 0,50 \mathrm{~m}$ en los bordes.

El tablero del puente principal se realizó por el sistema de voladizos sucesivos, procediendo simétricamente en cada pila. El vertido de hormigón se llevó a cabo por secciones de $3,40 \mathrm{~m}$, con el auxilio de cuatro cimbras móviles, al ritmo de $13,60 \mathrm{~m}$ por semana $(4 \times 3,40 \mathrm{~m})$.
En las ménsulas pretensadas se utilizaron barras de acero de alta resistencia $80 / 105$, de $26 \mathrm{~mm}$ de diámetro, con un espesor máximo de pretensado de 9.000 t. Las armaduras principales consisten en barras de $16 \mathrm{~mm}$ de diámetro.

Para la construcción de los viaductos de acceso se emplearon dos cimbras móviles - que eran verdaderas fábricas a pie de obra-, con una longitud de $50 \mathrm{~m}$ y un peso de $370 \mathrm{t}$, montadas sobre entramados metálicos de más de $80 \mathrm{~m}$ de longitud. Con ellas se realizaron los tramos al ritmo de uno cada 14 días. Una de las cimbras empezó en la isla de Svinö y la otra en la de Öland, para encontrarse en el centro del estrecho de Kalmar.

La capa de rodadura se compone de una capa de $12 \mathrm{~m}$
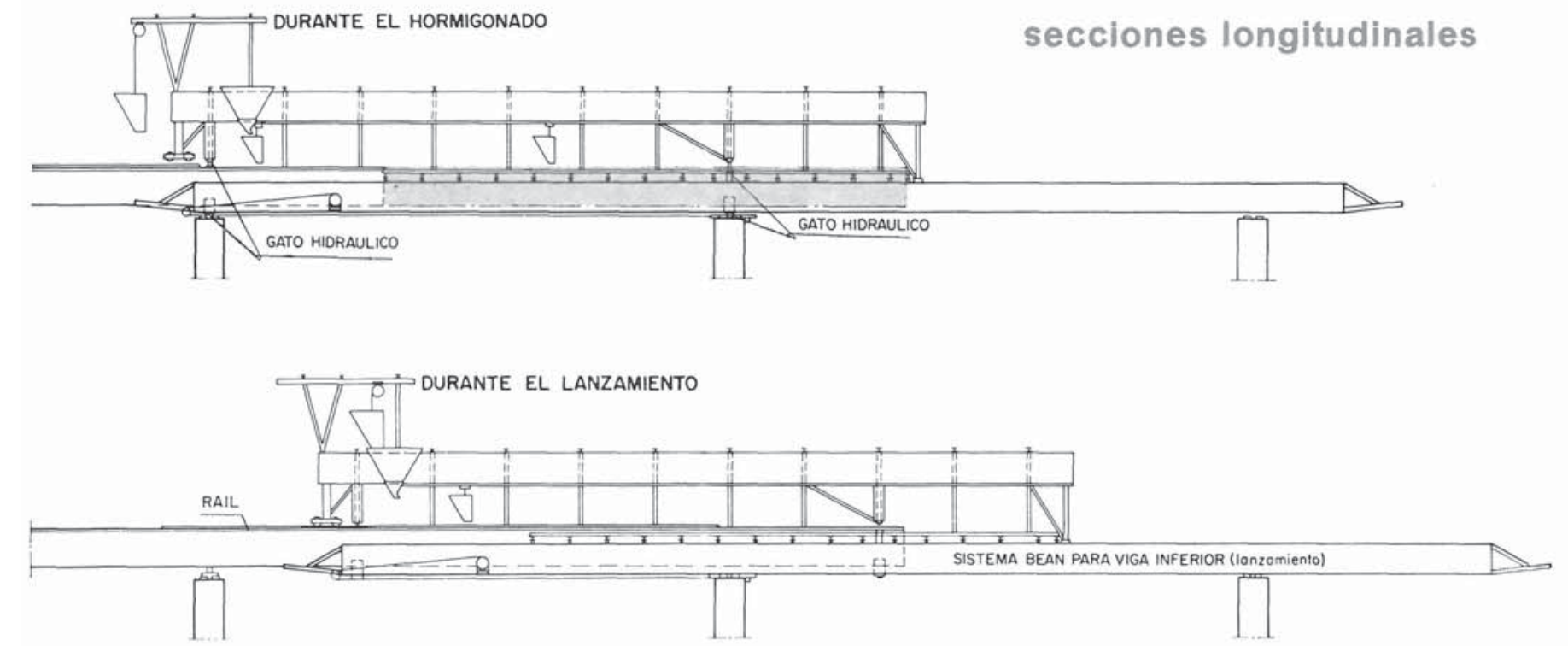

SECC. TRANSVERSAL-HORMIGONADO SISTEMA PARA VIGA SUPERIOR SECC. TRANSVERSAL-LANZAMIENTO
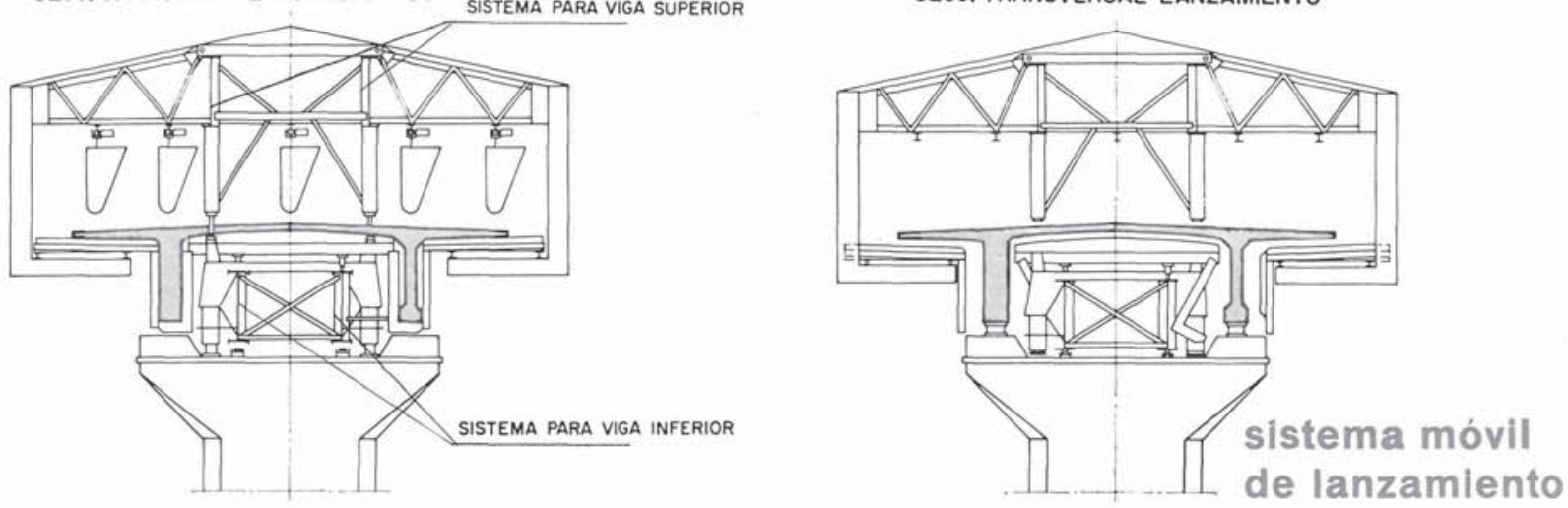

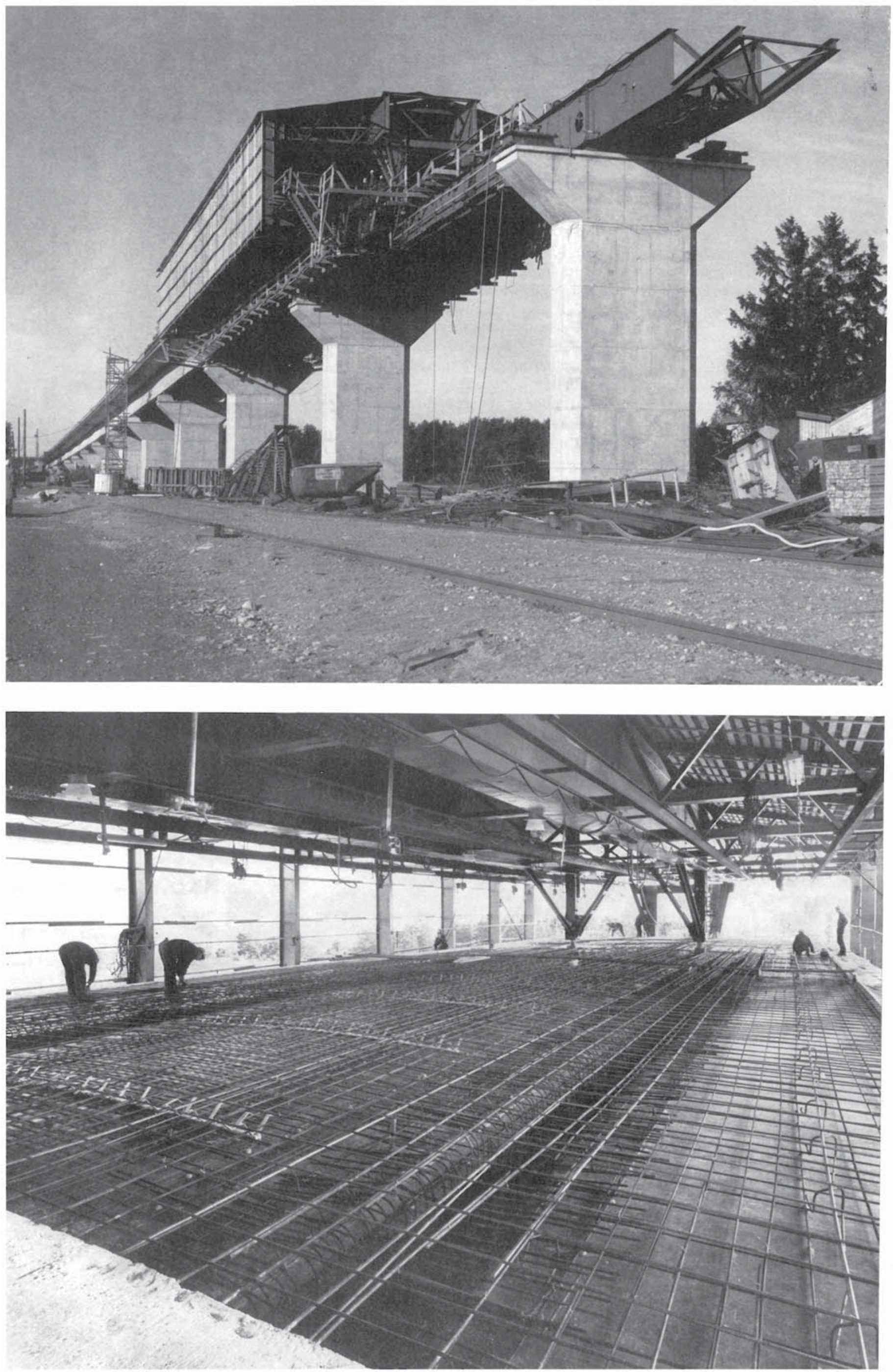


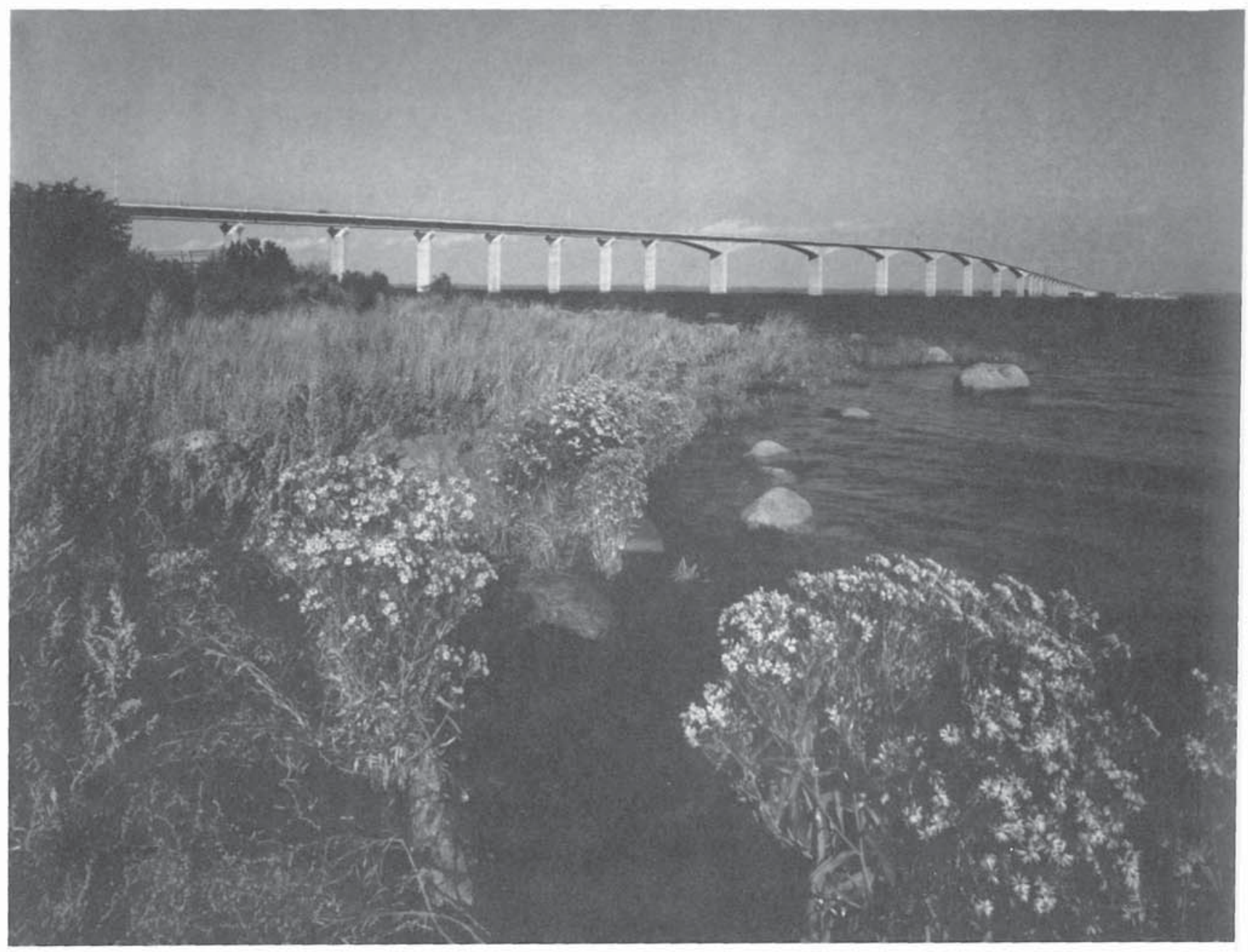

de fibra de vidrio, revestida de mástique asfáltico; una protección bituminosa de $10 \mathrm{~mm}$ de espesor; $35 \mathrm{~mm}$ de binder y $35 \mathrm{~mm}$ de capa de desgaste.
Se previeron sistemas de drenaje de agua y de evacuación de gases, mediante disposición de tubos de plástico con salidas por el intradós de los arcos.

La iluminación artificial fue especialmente cuidada y consiste en báculos de luces de $135 \mathrm{~W}$ de potencia, cada $35 \mathrm{~m}$ en el tablero, $y$ focos que iluminan las pilas del canal navegable.

Para la consideración de cargas admisibles y criterios de proyecto se adoptaron las normas suecas SOU 1961:12, además de tener en cuenta los esfuerzos del viento, las presiones de los bloques de hielo y las posibles colisiones con navíos.

En las tablas siguientes se relacionan los ensayos de resistencia a compresión y los esfuerzos admisibles.

El puente de Öland ha sido proyectado y realizado por la empresa Skånska Cementgjuteriet, por orden de la administración del Estado. 
TABLA I

RESULTADOS DE LOS ENSAYOS DE RESISTENCIA A COMPRESION A LOS 28 DIAS

\begin{tabular}{l|c|c|c|c|c|c|}
\hline Tipo de hormigón & $\begin{array}{c}\text { Número } \\
\text { de probetas }\end{array}$ & $\begin{array}{c}\text { Resistencia } \\
\text { media en } \\
\mathrm{kp} / \mathrm{cm}^{2}\end{array}$ & $\begin{array}{c}\text { Desviación } \\
\text { cuadrática } \\
\text { media }\end{array}$ & $\begin{array}{c}\text { Probetas con } \\
\text { resistencia } \\
\text { inferior al } \\
\text { minimo } \\
\text { autorizado }\end{array}$ \\
\hline K 300 vertido bajo el agua & 661 & 503 & 63 & 12,5 & - & - \\
\hline K 300 con aireante & 116 & 417 & 60 & 14,4 & 4 & 3,4 \\
K 300 & 749 & 397 & 66 & 16,6 & 19 & 2,5 \\
K 450 con aireante & 1.570 & 551 & 55 & 10,0 & 45 & 2,9 \\
\hline K 450 & 433 & 559 & 62 & 11,1 & 15 & 3,5 \\
\hline
\end{tabular}

TABLA II

ESFUERZOS ADMISIBLES EN EL HORMIGON EN CONDICIONES DE TRABAJO $\left(\mathrm{kp} / \mathrm{cm}^{2}\right)$

\begin{tabular}{l|c|c|c|c}
\hline \multicolumn{1}{r}{ Tipo de hormigón } & $\sigma_{\mathrm{k} 28}$ & $\sigma_{\mathrm{b}}$ & $\sigma_{\mathrm{bd}}$ & $\tau_{\mathrm{b}}$ \\
\hline K 450 & & & & \\
\hline K $450 \times$ c.a.p. & 450 & 140 & 36 & 9 \\
K 350 & 450 & 130 & 20 & - \\
K 300 & 350 & 110 & 32 & 8 \\
Vertido bajo el agua & 300 & 95 & 40 & 7.5 \\
\hline
\end{tabular}

$\sigma_{k s}=$ resistencia a los 28 dias en probetas cúbicas de $15 \mathrm{~cm}$ de lado

$\sigma_{\mathrm{b}}=$ resistencia a compresión en el ensayo a flexión

$\sigma_{\mathrm{bd}}=$ resistencia a tracción en el ensayo a flexión

$r_{\mathrm{b}}=$ resistencia a esfuerzo cortante

TABLA III

ESFUERZOS ADMISIBLES EN LAS ARMADURAS EN CONDICIONES DE TRABAJO $\left(\mathrm{kp} / \mathrm{cm}^{2}\right)$

\begin{tabular}{l|l|l|l}
\multicolumn{1}{c|}{ Armadura } & $\sigma_{\mathrm{s}}$ & $\sigma_{\mathrm{B}}$ & $\sigma_{\mathrm{a}}$ \\
\hline Barras deformadas & & & \\
\hline Ks $40 \quad \phi \leqslant 16 \mathrm{~mm}$ & 4000 & - & 2200 \\
Ks $40 \quad 16<\varnothing \leqslant 32 \mathrm{~mm}$ & 3800 & - & 2000 \\
Ks $60 \quad 0 \leqslant 16 \mathrm{~mm}$ & 6000 & - & 3000 \\
Barras de pretensado & & & \\
$\varnothing=26 \mathrm{~mm}$ & 8000 & 10500 & 6000 \\
\hline
\end{tabular}

$\sigma_{\mathrm{n}}=$ limite inferior de agotamiento

$\sigma_{1}=$ carga de rotura

$\sigma_{\mathrm{\alpha}}=$ tracciones admisibles 


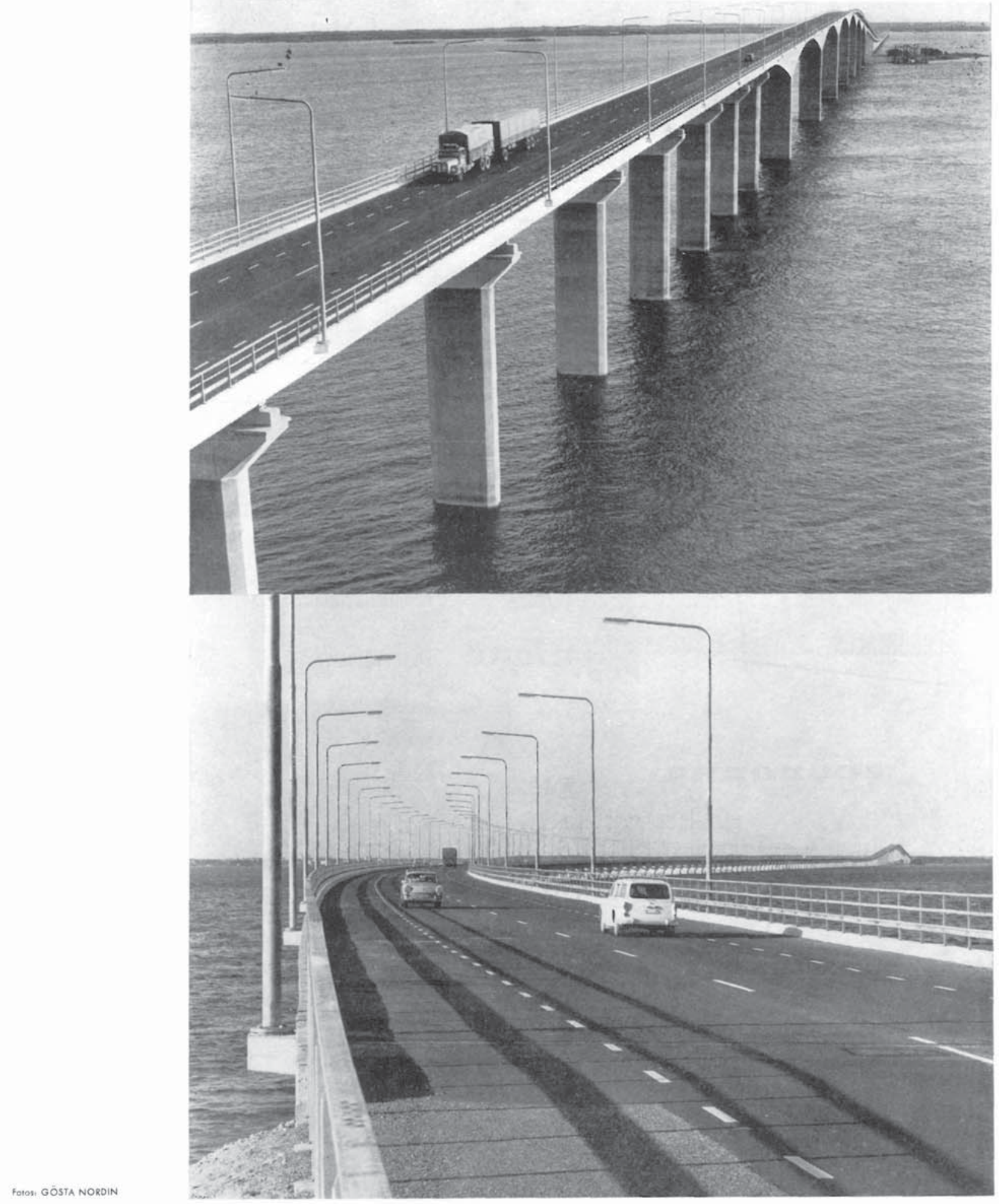

\section{résumé}

Le pont öland - Suède

Le pont öland franchit le détroit de Kalmar, partant de Möllstorpsläge, à l'île d'Öland, jusqu'à I'ile N. Skallö, d'ici à celle de Svinö et de celle-ci à Jutnabben, dans la péninsule suédoise. II a 155 travées: 147 de petite portée, deux de portée moyenne et six centrales, de $130 \mathrm{~m}$

Les arches dites hautes on été réalisées par le système d'encorbellements successifs et celles dites basses, par le lancement de grandes poutres en beton arme. Fondations sur pieux du coules in situ, en caissons viles pneta armé, exécutées à l'aide de coffrages glissants.

L'entreprise de construction a été Skanska Cementgiuteriet, pour le compte de l'Administration de l'Etat.

\section{summary}

The Öland bridge - Sweden

The öland bridge spans across the Kalmar strait, reaching from Möllstorpsläge, on the island of Oland, to the island of N. Skallö; from here to the island of Svinö and then to Jutnabben, on the Swedish peninsula. It has 155 spans out of which 147 are small spans, $130 \mathrm{~m}$, with a total length of $6.070 \mathrm{~m}$.

The high arches have been constructed by the system of successive corbels and the low ones with a launching of big beams of rein. forced concrete. Foundations on columns or poured on site in pneumatic emptied boxes. depending on the cases and piers of reinforced concrete, constructed by means of sliding

The Construction Firm has been Skanska Cementgjuteriet on account of the Government Administration.

\section{zusammenfassung}

Die Öland Brücke - Schweden

Die Öland Brucke streckt sich über die Kalmar Meerenge, von Möllstorpsläge, auf der Insel Oland bis die Insel N. Skallö; von dort aus bis die Insel Svinö und von dieser bis Jutnab. ben auf der schwedischen Halbinsel. Sie hat 155 Bogenoffnungen wovon 147 kleine Bogen jeder $130 \mathrm{~m}$, mit einer Gesamtlänge von $6.70 \mathrm{~m}$. Die hohen Bogen sind mit dem System von
aufeinanderfolgenden Auskragungen und die aufeinanderfoigenden Auskragungen und die ausgeführt. Gründungen auf Säulen, oder in pneumatisch geleerten Kasten auf der Stelle geschuttet je nach dem Fall und Pfeiler aus Stahlbeton, mit einer Gleitschalung konstruiert. 